\title{
The application of magnetic self-filter to optimization of AIN film growth process during the impulse plasma deposition synthesis
}

\author{
RAFAL CHODUN $^{1 *}$, KATARZYNA NOWAKOWSKA-LANGIER $^{2}$, \\ SEbastian OKRASA ${ }^{1}$, KRZYSZTOF ZdUNEK ${ }^{1}$ \\ ${ }^{1}$ Faculty of Materials Science and Engineering, Warsaw University of Technology, Woloska 141, 02-507 Warsaw, Poland \\ ${ }^{2}$ National Centre for Nuclear Research (NCBJ), Andrzeja Soltana 7, 05-400 Otwock-Swierk, Poland
}

\begin{abstract}
This work presents the very first results of the application of plasma magnetic filtering achieved by a coil coupled with an electrical circuit of a coaxial accelerator during the synthesis of AlN thin films by use of Impulse Plasma Deposition method (IPD). The uniqueness of this technical solution lies in the fact that the filter is not supplied, controlled and synchronized from any external device. Our solution uses the energy from the electrical circuit of plasma accelerator. The plasma state was described on the basis of OES studies. Estimation of the effects of plasma filtering on the film quality was carried out on the basis of characterization of structure morphology (SEM), phase and chemical composition (vibrational spectroscopy). Our work has shown that the use of the developed magnetic self-filter improved the structure of the AlN coatings synthesized under the condition of impulse plasma, especially by the minimization of the tendency to deposit metallic aluminum droplets and columnar growth.
\end{abstract}

Keywords: IPD method; magnetic filter; nanocrystalline films; thin films

(C) Wroclaw University of Technology.

\section{Introduction}

Nowadays, there is a noticeable increase in the interest in aluminum nitride (AIN) in electronics and optoelectronics. This material is a very special compound which combines various sets of properties. AlN is a dielectric material with a wide direct band gap of about $6 \mathrm{eV}$ [1-3], which stands out among the oxides, carbides and nitrides with good thermal conductivity of about $285 \mathrm{~W} \cdot \mathrm{m}^{-1} \cdot \mathrm{K}^{-1}$ [4]. A set of properties that represent this film material makes it appropriate for a wide range of applications in the technology of electronic materials [510]. Encouraging opportunities that entail the development of an efficient synthesis technology of AlN has led to numerous publications. Among a variety of synthesis technologies, such as sintering methods [11], CVD [12, 13] and PVD [14, 15], the latter have become the leading methods, mostly

*E-mail: rafal.chodun@inmat.pw.edu.pl because of the ability of synthesis of relatively slightly heated substrates, chemical purity, and cost effectiveness. An important disadvantage of these methods is the presence of metallic droplets, such as phases in the structure of the films, incomplete reaction of substrates as a result of an insufficient ionization degree of reactants, porous, conical or columnar structure of the deposited films.

We have set ourselves the objective of developing an efficient technology of aluminum nitride films synthesis while minimizing the drawbacks mentioned above, characteristic of the surface engineering methods. For this purpose we have chosen the IPD method (Impulse Plasma Deposition) as, in our view, it is the one which guarantees an effective synthesis of this nitride. The IPD method by using strong non-equilibrium impulse plasma allows the use of non-conventional means of synthesis, taking into account the parameters such as, for example, electrical energy resulting from participation of charged particles and electric field. The importance 
of electrical energy is very strongly manifested in this method, the most among all other methods of plasma surface engineering. The examples of the effects of such a synthesis are the phases characterized by high barriers of nucleation and metastable phases [16-18].

The reasons for choosing the IPD as the method of AlN films synthesis for the experiment are encouraging, however, the undoubted drawback of this method is a high probability of reaching the electrode material of plasma accelerator in the form of metallic droplets to the substrate, together with vapors of synthesized material. This phenomenon occurs due to the relatively low melting point of aluminum of which the accelerator is made. In order to get rid of the droplets we have developed a magnetic self-filter. The plasma magnetic filters became permanently applicable in the films deposition methods, in which there is an awareness of the occurrence of metallic droplets [19-21]. An element of novelty in our development is that the filter is directly supplied with the current from the plasma accelerator due to the high current flowing between the electrodes of the accelerator during the discharge. The characteristic feature of our development let us avoiding further problems with an appropriate supply in power and synchronization between the filter current peaks and the plasma packs, which have a lifetime of tens of $\mu$ s.

\section{Experimental}

The scheme of the apparatus used in the experiment is presented in Fig. 1A. Impulse plasma is generated in the accelerator which consists of coaxially arranged electrodes made of aluminum ( $99.5 \%$ purity). Nitrogen was used as a working gas, individual doses of which were injected by impulse valve into the interelectrode space of the accelerator. The impulse plasma is initiated between the electrodes of the accelerator as a result of high current discharge of $2 \mathrm{mF}$ capacitors with $1 \mathrm{kV}$ voltage. After that, the plasma is accelerated by Ampere force towards the accelerator outlet in a form of paraboloidal plasmoid. The electrodes material is continuously eroded, and the products of erosion enrich the plasma as synthesis reactants. A precise characterization of the IPD method has been described in the literature [22, 23]. Fig. 1B shows the wiring diagram of the magnetic filter. Fig. 1C presents a photograph of the filter installed in the vacuum chamber. The filter is made of a bent copper bar in a form of a coil which is connected with an electric circuit of accelerator in series. As presented in the diagram, during the first half of the discharge waveform, the internal electrode gets a positive bias. The electric current flows through the plasma in the direction of external electrode, and then along the turns of the coil, and thus generates the magnetic field whose induction vector is directed along the $\mathrm{z}$-axis of accelerator.

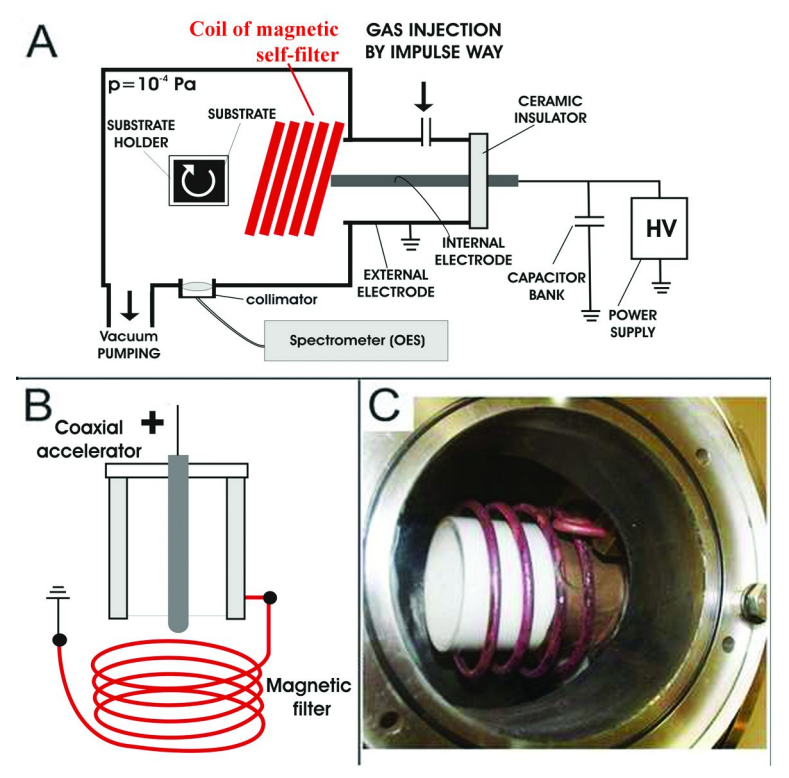

Fig. 1. Schematic diagram of the apparatus used in the experiment (A), wiring diagram of the coil connected with plasma accelerator (B) and photograph of the coil coupled with accelerator installed in the vacuum chamber.

Two processes of AlN films deposition were carried out: under the conditions of plasma filtering and with the use of a standard version of accelerator (without magnetic filter). The layers were deposited on silicon substrates oriented perpendicularly to the z-axis of accelerator. Synthesis processes were carried out in a time of 5000 impulses generated at a frequency of $0.2 \mathrm{~Hz}$. In the case of the standard process, the substrate was placed in the 
axis of the accelerator at a distance of $250 \mathrm{~mm}$, thus the substrate surface was exposed to all products of accelerator erosion. During the process of synthesis with the magnetic filtering, the coil was bent at an angle of $45^{\circ}$ and the substrate was placed at the outlet of the coil, positioned parallel to the plane of the last turn of the coil.

During the experiment, two optical spectra of filtered and unfiltered impulse plasma were recorded. OES measurements were performed by means of a Mechelle ${ }^{\circledR} 900$ spectrometer operated in the wavelength range of $350 \mathrm{~nm}$ to $650 \mathrm{~nm}$ with the exposition time equal to $200 \mu \mathrm{s}$. A freepropagating plasma was registered by the optical collimator placed at a distance of $250 \mathrm{~mm}$ from the accelerator outlet, perpendicular to the streamer.

Deposited on silicon substrates AlN films were examined by SEM in terms of morphology of the structures with a ZEISS ULTRA Plus device. The samples were observed at a $45^{\circ}$ angle. An estimation of the quality of the synthesized film surfaces in order to detect the metallic droplets, was carried out by an optical microscope. Micro Raman studies of the synthesized layers were performed using the JASCO NRS-5100 device with Ar+ laser of $2.33 \mathrm{eV}(532 \mathrm{~nm})$ excitation energy. Meanwhile, vibrational spectra registering the $6.5 \mathrm{~mW}$ laser beam was focused onto $10 \mu \mathrm{m}$ spots.

\section{Results and discussion}

Fig. 2 shows a photograph of a single shot of the plasma accelerator taken at a long exposition time of the camera perpendicular to the accelerator axis. In order to take a picture (for presentation purposes) the filter's coil was bent down. In the picture, the location of the accelerator, which was hidden behind the vacuum chamber wall, and filter bending radius were schematically indicated. This photograph confirms the practical work of our invention. The plasma pack generated in the coaxial plasma accelerator is ducted inside the bent coil, preserving its curvature. As a result of the working gas injection into the interelectrode space, the discharge between electrodes is triggered. The generated current flows through the magnetic filter and generates a magnetic field with an induction vector along the direction of the filter axis. The generated magnetic field influences the transport conditions of plasma which aligns along the direction of the magnetic field lines. Moreover, in comparison with plasma generated in a standard accelerator, impulse plasma is more dense and focused in a form of a stream as a result of the bias sheath of the coil. To study the filtered plasma state, we used OES technique. Fig. 3 presents a comparison of registered optical spectra of free-propagating plasma stream during the process of generation of impulse plasma under standard conditions and with the use of magnetic filtering.

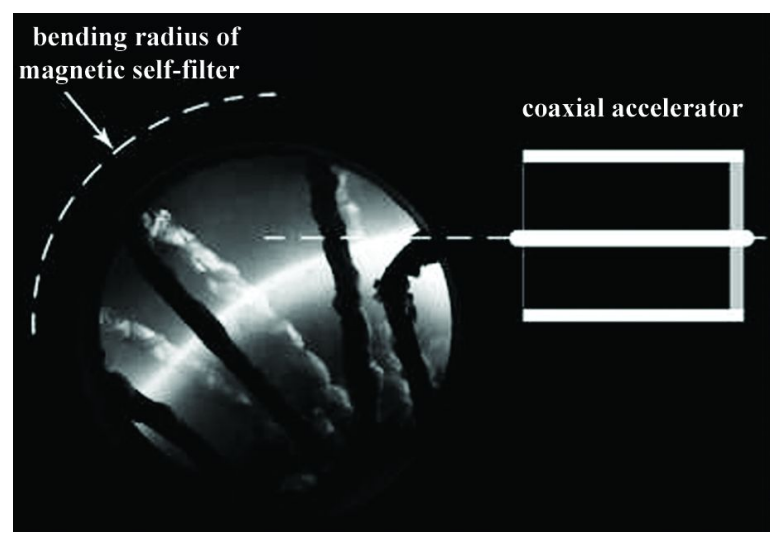

Fig. 2. Photograph of full single plasma pack guided by the bent down coil of filter.

From the obtained spectra, we can conclude that impulse plasma has a high ionization degree. The following components have been indicated:

- ionizes nitrogen species originating from excitation of working gas particles (N II, N III, N V);

- ionizes aluminum species originating from erosion and excitation of material of electrodes (Al II, Al III, Al IV);

- hydrogen and carbon species originating probably from erosion of insulating polymer parts of the vacuum chamber.

The spectra registered while the plasma accelerator was coupled with the coil are more intense than the others. In the spectra obtained while the coil 


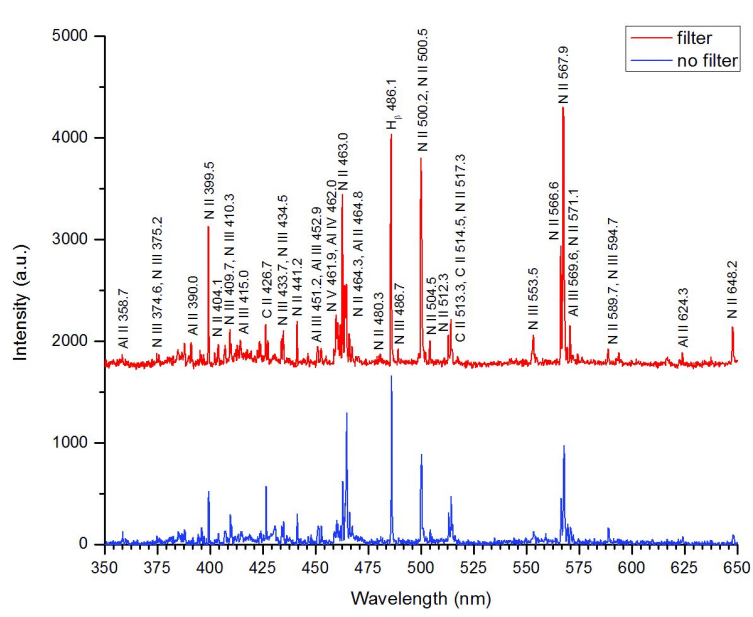

Fig. 3. Comparison of optical spectrum of magnetically filtered impulse plasma (red) with optical spectrum of plasma generated at standard apparatus of IPD method (blue).

is on, some extra spectra lines appear (for instance: N III 374.6, N III 375.2, N III 486.7, N III 594.7). It seems that the application of the filter may slightly increase the ionization state of impulse plasma. It is well known that applying a magnetic field forces causes the plasma electrons to move along helical paths around magnetic lines, which results in decreasing the free path of electrons. This phenomenon is widely used in plasma surface engineering methods cause it provides the efficient energy transfer from the source to the plasma state. We are convinced by the obtained results that the additive energy of magnetic field created by the coil enhanced the energetic state of impulse plasma so we tried to find out how this influenced the product of synthesis.

Vibrational spectroscopy studies were used to identify the product of the synthesis. Fig. 4 shows the measured Raman spectra with extracted AlN phonon components. The peak registered at $\sim 520 \mathrm{~cm}^{-1}$ comes from silicon substrate. In both cases, the AlN film synthesized with plasma accelerator in the standard version and the film synthesized using the magnetic filter, we indicated the presence of hexagonal AlN phonon modes, reported in the literature as: $\mathrm{n}_{\mathrm{TO}}\left(\mathrm{A}_{1}\right) 607$ to $614 \mathrm{~cm}^{-1}, \mathrm{n}^{(2)}\left(\mathrm{E}_{2}\right) 655$ to $660 \mathrm{~cm}^{-1}$ [24]. AlN

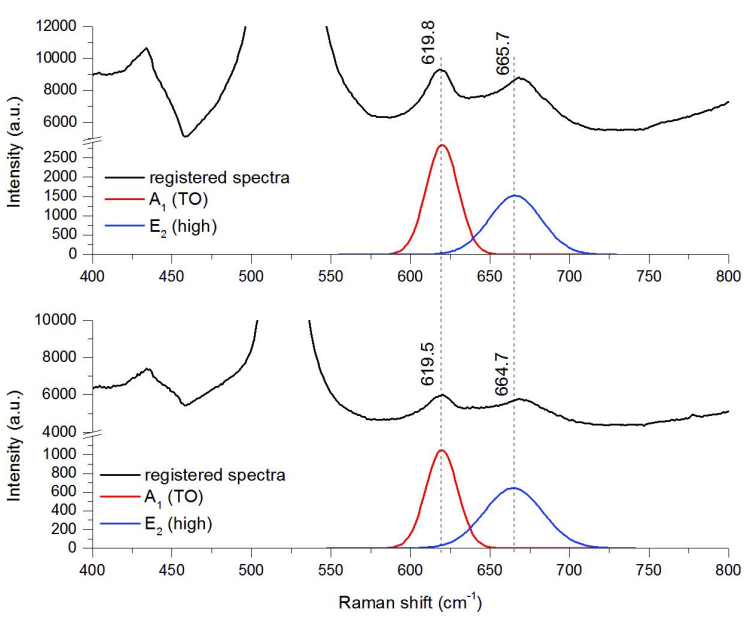

Fig. 4. Raman spectra of AlN films deposited on silicon substrates during the experiment with magnetic filtering (top) and without filtering (bottom).

peaks in both spectra are almost in the same positions. What is noticeable is that the spectrum of the film deposited in the not filtered process has a slightly lower ratio of AlN peaks signal to fluorescence what may be caused by the existence of metallic fraction in the deposited film (strong fluorescence of metals at visible light).

The main aim of our study was to reduce the content of the metallic phase occurring in the form of droplets of aluminum in the structure of AlN films deposited on substrates. Fig. 5 compares the photographs of the film surfaces obtained by using the accelerator in two variants: without the magnetic plasma filter and with the filter. Relatively big metallic macroparticles were indicated by arrows. From a comparison of the images, it is clear that the synthesis system with the filter applied favors obtaining droplets-free films. Our system works as a kind of plasma component separator where the criterion of separation is the mass of the molecules. Light components characterized by molecular dispersion are effectively guided by the filter and delivered to the substrate. A heavy fraction, created during melting the aluminum electrodes by arc discharges and then by the erosion through acceleration of the plasmoids moving towards accelerator outlet, is exported out of range of the filter and is not delivered to the substrate. 


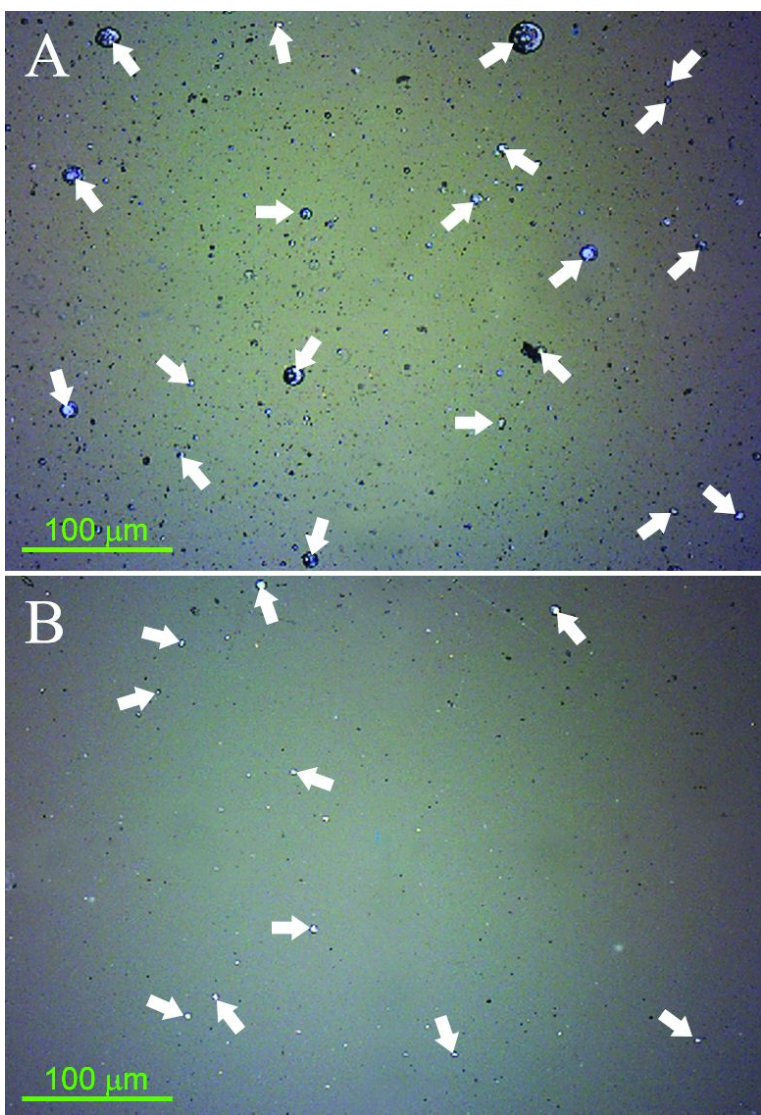

Fig. 5. Surface images of AlN films deposited by a standard apparatus (A) and by the apparatus with the filter coupled with accelerator.

Photographs of the AlN film surfaces observed by an optical microscope provide information about the effect of plasma filtering on the film structure. In order to estimate the films morphology the SEM technique was used. Fig. 6 presents the results of the structural studies. On the surface of the film obtained in the standard version of accelerator (without filter) the characteristic metallic droplets can be noticed, which confirms the conclusions from the analysis obtained using optical microscopy and Raman spectroscopy. Interestingly, the use of a magnetic filter of impulse plasma affects the structure of the aluminum nitride films. The film obtained with the plasma filtering process is characterized by smaller crystallites than the films synthesized using the standard accelerator. Furthermore, this layer is two-fold thinner. Taking into account that the photographs

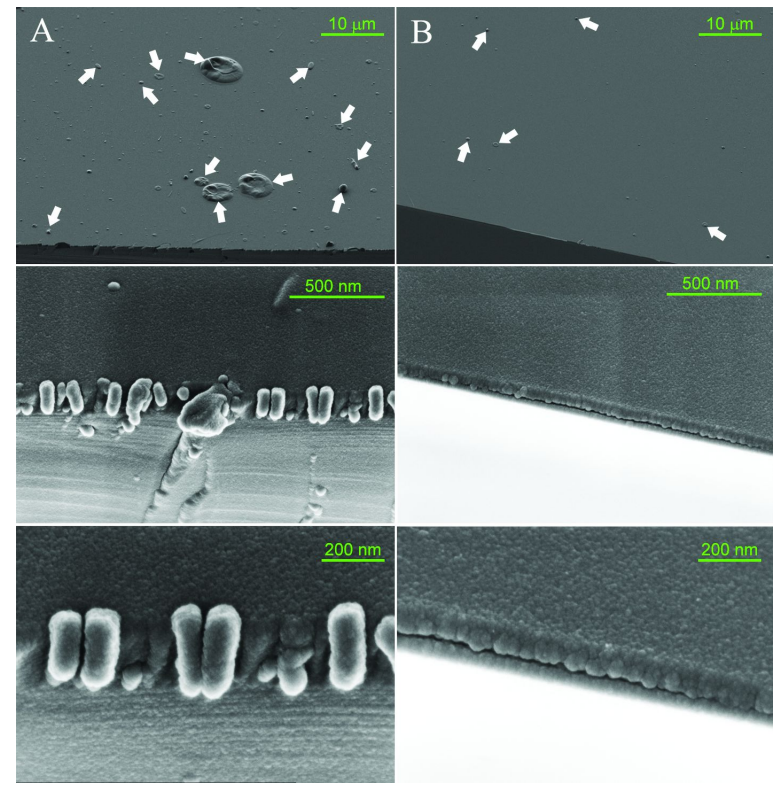

Fig. 6. SEM photographs of AlN films structures deposited during the processes with the standard accelerator (left column) and using a plasma filtering (right column).

were taken at a $45^{\circ}$ angle, the thickness of the films can be estimated as $300 \mathrm{~nm}$ for the standard IPD process and $150 \mathrm{~nm}$ for the IPD process with filtered plasma. This fact arouse suspicion that the cause of the thinner film is the orientation of the substrate surface which was arranged at an angle of $45^{\circ}$ and shifted off the accelerator axis, (which lowered the deposition kinetics) However, the direction of growth of the crystallites indicates that the plasma components were supplied from the direction normal to the substrate surface as the crystallites are oriented perpendicular to the substrate surface. Returning to the results obtained during the OES and structural studies we suppose that the structural differences (changes in morphology from columnar into equiaxed grained structure and growth kinetics from 0.6 to $0.3 \AA$ per single power impulse) are probably an effect of higher energy of plasmoids, additionally enhanced by the magnetic filter. It has been reported in literature, that the growth of coatings, deposited under the conditions of high ion flux or from the plasma whose components are characterized by relatively high kinetic energy, is strongly affected by the interaction 
of impinging energetic species. Anders extended the well-known models of film growth on the range of more energetic species [25]. In the Anders model of a film growth, the range of existence of nano- and amorphous structures with relatively lower kinetics of growth is indicated. We suppose that conditions of our experiments placed the obtained AlN film structures in that specific region and all the revealed structural differences are included in Anders model. The IPD process realized with the magnetic filter enhanced the energy of plasma species, so the growing coating was sputtered slightly more efficiently by the more energetic plasma ions. On the other hand, more energetic species created defects in growing films and that probably explains the achieved dense nanostructure.

\section{Conclusions}

In this work, we have presented a concept of the use of impulse plasma magnetic filtration by a unique self-developed magnetic filter, coupled directly to the coaxial plasma accelerator, which works without any external power supply, but is controlled and synchronized with the source of the plasma. Our work has shown that the developed filter fulfills the expected objective of reducing the content of metallic phase in the structure of the films. Moreover, our studies indicate that the usage of such a development changes the conditions of film growth. OES studies suggest that the synthesis environment is characterized as more energetic. In this case, the growth of films occurs in different conditions which favor the ultra fine and dense structure of deposited films.

\section{Acknowledgements}

This work was supported by the Polish State National Science Centre within the Project 2013/09/B/ST8/02418.

\section{References}

[1] Guo Q., Yoshida A., Jpn. J. Appl. Phys., 33 (1994), 2453.

[2] Teisseyre H., Perlin P., Suski T., Grzegory I., Porowski S., Jun J., Pietraszko A., Moustakas T. D., J. Appl. Phys., 76 (1994), 2429.
[3] Yamashita H., Fukui K., Misawa S., Yoshida S., J. Appl. Phys., 50 (1979), 896.

[4] Slack G.A., Tanzilli R.A., Pohl R.O., VanderSANDE J.W., J. Phys. Chem. Solids, 48 (1987), 641.

[5] Chiu K.H., Chen J.H., Chen H.R., Huang R.S., Thin Solid Films, 515 (2007), 4819.

[6] Yang R.Z., Hsiung C.M., Chen H.H., Wu H.W., ShIH M.C., Microwave Optic. Techn. Lett., 50 (2008), 2863.

[7] Bose S., Mazumder S.K., Solid State Electron., 62 (2011), 5.

[8] Kelekci O., Tasli P., Cetin S.S., Kasap M., Ozcelik S., Ozbay E., Curr. Appl. Phys., 12 (2012), 1600 .

[9] Iborra E., Olivares J., Clement M., Vergara L., SAnz-Hervás A., SAngrador J., Sensor. Actuat. A-Phys., 115 (2004), 501.

[10] Belyanin A.F., Bouilov L.L., Zhirnov V.V., Kamenev A.I., Kovalskij K.A., Spitsyn B.V., Diam. Relat. Mater., 8 (1999), 369.

[11] QiU J.Z., HotTa Z., Watari K., Mitsuishi K., YAMAZAKi M., J. Eur. Ceram. Soc., 26 (2006), 385.

[12] Fischer R.A., Miehr A., Ambacher O., MetZGER T., Born E., J. Cryst. Growth, 170 (1997), 139.

[13] Tanaka Z., Hasebe Z., Inushima T., Sandhu A., OHOYA S., J. Cryst. Growth, 209 (2000), 410.

[14] Dimitrova V., Manova D., Paskova T., Uzunov T., IVAnov N., DecheV D., Vacuum, 51 (1998), 161.

[15] Manova D., Dimitrova V., FukareK W., KarpuzOV D., Surf. Coat. Tech., 106 (1998), 205.

[16] NOWAKOWSKA-LANGIER K., WIERZBińsKi E., ZduneK K., Kopcewicz M., Vacuum, 77 (2005), 287.

[17] Nowakowska-Langier K., Chodun R., NIetubyc R., Minikayev R., Zdunek K., Appl. Surf. Sci., 275 (2013), 14.

[18] NowakowsKa-LAngier K., ZdUneK K., Wierzbinski E., Surf. Coat. Tech., 204 (2010), 2564.

[19] Anders S., Anders A., Brown I.G., J. Appl. Phys., 74 (1993), 4239.

[20] Anders A., Surf. Coat. Tech., 120 - 121 (1999), 319.

[21] Zimmer O., Surf. Coat. Tech., 200 (2005), 440.

[22] ZduneK K., Surf. Coat. Tech., 201 (2007), 4813.

[23] ZdUNEK K., NOWAKOWSKA-LANGIER K., ChOdUn R., KUPCZYK M., SIWAK P., Vacuum, 85 (2010), 514.

[24] Collins A.T., Lightowlers E.C., Dean P.J., Phys. Rev., 158 (1967), 833.

[25] Anders A., Thin Solid Films, 518 (2010), 4087. 